\title{
Bird Observations in Severnaya Zemlya, Siberia
}

\author{
J. DE KORTE ${ }^{1}$, A.E. VOLKOV² and M.V. GAVRILO ${ }^{3}$
}

(Received 22 April 1994; accepted in revised form 9 February 1995)

\begin{abstract}
Fieldwork in different parts of Severnaya Zemlya in 1985, 1991, 1992 and 1993 and aerial surveys in 1994 revealed a limited bird fauna with a total of 17 breeding species. The most numerous breeding birds are cliff-nesting seabirds, comprising little auk (Alle alle), 10 000-80 000 pairs; kittiwake (Rissa tridactyla), 5000-10 000; black guillemot (Cepphus grylle), 10005000; ivory gull (Pagophila eburnea), 1000-2000; and glaucous gull (Larus hyperboreus), 500-1000. They breed all over the archipelago, usually in rather small mixed- or single-species colonies. Arctic tern (Sterna paradisaea) 100-500, and herring gull (Larus argentatus) 1-10, breed as solitary pairs or with a few pairs together. Of tundra birds, only brent goose (Branta bernicla), purple sandpiper (Calidris maritima) and snow bunting (Plectrophenax nivalis) are found breeding on most of the major islands. The other tundra species—red-throated diver (Gavia stellata), king eider (Somateria spectabilis), sanderling (Calidris alba), Arctic skua (Stercorarius parasiticus), long-tailed skua (Stercorarius longicaudus), snowy owl (Nyctea scandiaca) and Lapland bunting (Calcarius lapponicus) — breed in small numbers and in limited areas, often not every year.

Of the even fewer mammal species, reindeer (Rangifer tarandus) occurs occasionally, while Arctic fox (Alopex lagopus) and collared lemming (Dicrostonyx torquatus) are locally common in some years.
\end{abstract}

Key words: Severnaya Zemlya, high Arctic birds, breeding distribution

\begin{abstract}
РЕФЕРАТ. Полевые исследования в разлячных частях архипелага Северная Земия в 1985, 1991, 1992, 1993 годах и обследование с вертолета в 1994 году показалн, что орнитофауна этого архнпелага бедна и вкмючает только 17 видов гнездящихся птиџ. Нанболее мнопочнсленны морские колоннальные птиды: мюрик (Alle alle) $10000-80000$ пар, моевка (Rissa tridactyla) $5000-10000$, чистик (Cepphus grylle) $1000-5000$, белая чайка (Pagophila ebuтnеa) 1000 - 2 000, бургомистр (Larus hyperboreus) 500 - 1000. 100 - 500 пар помярной крачки (Sterna paradisaea) и 1 - 10 nap серебристой чайкн (Larus argentatus) гнездятся вне колоний иин небольшими группамн. Из тундровых птиц только черная казарка (Branta bemicla), морской песочник (Calidris maritima) и пуночка (Plectrophenax nivalis) гнездятся на большинстве островов. Доугие тундровые виды: краснозобая гагара (Cavia stellata), гага-гребенушка (Somateria spectabilis), песчанка (Calidris alba), короткохвостый поморняк (Stercorarius parasiticus), дияннохвостый поморник (Stercoratius longicaudus), белая сова (Nyctea scandiaca) и мапиандский подорожник (Calcarius lapponicus) тнездятся в небольшом количестве на ограниченных территориях, обычно не каждый год. Приведены также краткие сведения о наземных ммекопитаюџих архнпелага: размножаюџихся - дикий северный олень (Rangifer tarandus), песей (Alopex lagopus), копытный Аемминг (Dicrostonyx torquatus).
\end{abstract}

RÉSUMÉ. Des études sur le terrain menées dans différentes parties de la Severnaïa Zemlia en 1985, 1991, 1992 et 1993 ainsi que des relevés aériens effectués en 1994 ont révélé une avifaune limitée comptant au total 17 espèces reproductrices. Les oiseaux nicheurs les plus nombreux sont ceux nichant sur les falaises. Ils comprennent le mergule nain (Alle alle) [10 000 à 80000 paires]; la mouette tridactyle (Rissa tridactyla) [5000 à 10 000]; le guillemot à miroir (Cepphus grylle) [1000 à 5000]; la mouette blanche (Pagophila eburnea)[1000 à 2000]; et le goéland bourgmestre (Larus hyperboreus) [500 à 1000]. Ils nichent sur tout l'archipel, en général en colonies plutôt petites rassemblant des espèces mixtes ou une seule espèce. La sterne arctique (Sterna paradisaea) [100 à 500], et le goéland argenté (Larus argentatus) [1 à 10], nichent en paires solitaires ou avec quelques autres paires. Des oiseaux de la toundra, seuls la bernache cravant (Branta bernicla), le bécasseau violet (Calidris maritima) et le bruant des neiges (Plectrophenax nivalis) nichent sur la plupart des grandes îles. Les autres espèces de la toundra - le huart à gorge rousse (Gavia stellata), l'eider à tête grise (Somateria spectabilis), le bécasseau sanderling (Calidris alba), le labbe parasite (Stercorarius

\footnotetext{
${ }^{1}$ Arctic Centre, University of Groningen, Oude Kijk in 't Jatstraat 26, Postbus 716, 9700 AS Groningen, The Netherlands

${ }^{2}$ All-Russian Research Institute of Nature Conservation and Reserves (Vserosiyskiy nauchno-issledovatel'skiy Institut okhrany prirody i zapovednogo dela [VNII priroda]), 113628 Moscow, Znamenskoe-Sadki, Russia

${ }^{3}$ The Arctic and Antarctic Research Institute (Arkticheskiy i Antarkticheskiy nauchno-issledovatel'skiy Institut, [AANII]), 119220, St. Petersburg, ulitsa Beringa 38, Russia

(c) The Arctic Institute of North America
} 
parasiticus), le labbe à longue queue (Stercorarius longicaudus), le harfang des neiges (Nyctea scandiaca) et le bruant lapon (Calcarius lapponicus) — nichent en petits nombres et dans des zones limitées, et souvent pas chaque année.

Parmi les espèces de mammifères — encore moins nombreuses que les oiseaux —on trouve de temps à autre le renne (Rangifer tarandus), tandis que le renard arctique (Alopex lagopus) et le lemming à collerette (Dicrostonyx torquatus) abondent localement certaines années.

Mots clés: Severnaïa Zemlia, oiseaux de l'Extrême-Arctique, distribution des oiseaux nicheurs

Traduit pour la revue Arctic par Nésida Loyer.

\section{INTRODUCTION}

The archipelago of Severnaya Zemlya is of zoogeographical interest as it is situated between the western and eastern Russian Arctic, separated from the Taymyr Peninsula by a wide strait. Until recently we knew little about the occurrence and breeding distribution of birds in this archipelago. It is the least surveyed of the Russian high-arctic archipelagos, which include Frans Josef Land, New Siberian Islands, Wrangel Island and Herald Island.

Severnaya Zemlya was discovered in 1913 by the expedition of Vil'kitskiy (Nasonov, 1916; Starokadomskiy, 1946; Starkov, 1985). It was first described by the geologist Urvantsev (1935), who had his base in A. Sedova (A. = Arkhipelag) and worked in the whole archipelago from 1930 to 1932 . Both Urvantsev (1935) and Ushakov (1951) reported information about animals hunted on the main islands. The ornithologist Demme (1934) also worked in A. Sedova from 1932 to 1934 . From 1935 until 1937, there was a weather station at M. Olovyannyy (abbreviations of geographical names in Table 1). From 1937 until 1953 there was a meteorological station on O. Domashniy (Kharitonovich, 1940). In 1953 it was moved to O. Golomyannyy where it remained until it was closed in 1994. On O. Bol'shoy a weather station was operated from 1940 to 1991. On O. Malyy Taymyr and at B. Solnechnaya and M. Peschannyy, weather stations were operating from 1943, 1951 and 1961 respectively until they were closed in 1994. All these stations carried out some ornithological programmes (Uspenskiy, 1969). In 1981 a scientific station of the Arctic and Antarctic Institute (AANII) in St. Petersburg was created at M. Vatutina, from where it was moved to a site near M. Baranova in 1988. In 1991 BARC-Company (St. Petersburg) privatised this station and gave it the name $\mathrm{S}$. Prima; today it is a centre of scientific and tourist activities.

Miscellaneous data about animals are presented by the geographer Semenov (1971) who worked on O. Oktyabr'skoy Revolutsii in 1950 with the geographer E.S. Korotkevich (AANII, unpubl. data). From 1974 to 1981, V.M. Makeev (AANII, unpubl. data) worked inland on this island. In 1982 Bulavintsev (1984) did some bird observations in O. Bol'shevik. In 1982 Belikov worked in the south of O. Bol'shevik, and in 1983 Belikov and Randla worked in A. Sedova and O. Oktyabr'skoy Revolutsii (Belikov, 1987; Belikov and Randla, 1987). Ornithological expeditions were done by the authors on O. Oktyabr'skoy Revolutsii in 1985 and on O. Bol'shevik in 1991 and 1992. General surveys by helicopter through the whole archipelago were done in 1993 and 1994. Only a few ornithological observations have been published, mostly by people with a primary interest other than birds; they are mostly anecdotal and all are in Russian. In this paper we summarize the published and unpublished data of previous expeditions and those of our expeditions to add to our knowledge of the distribution and ecology of high-arctic birds in some of the least known arctic islands of the world. Common and scientific bird names and their sequence conform to Voous (1977).

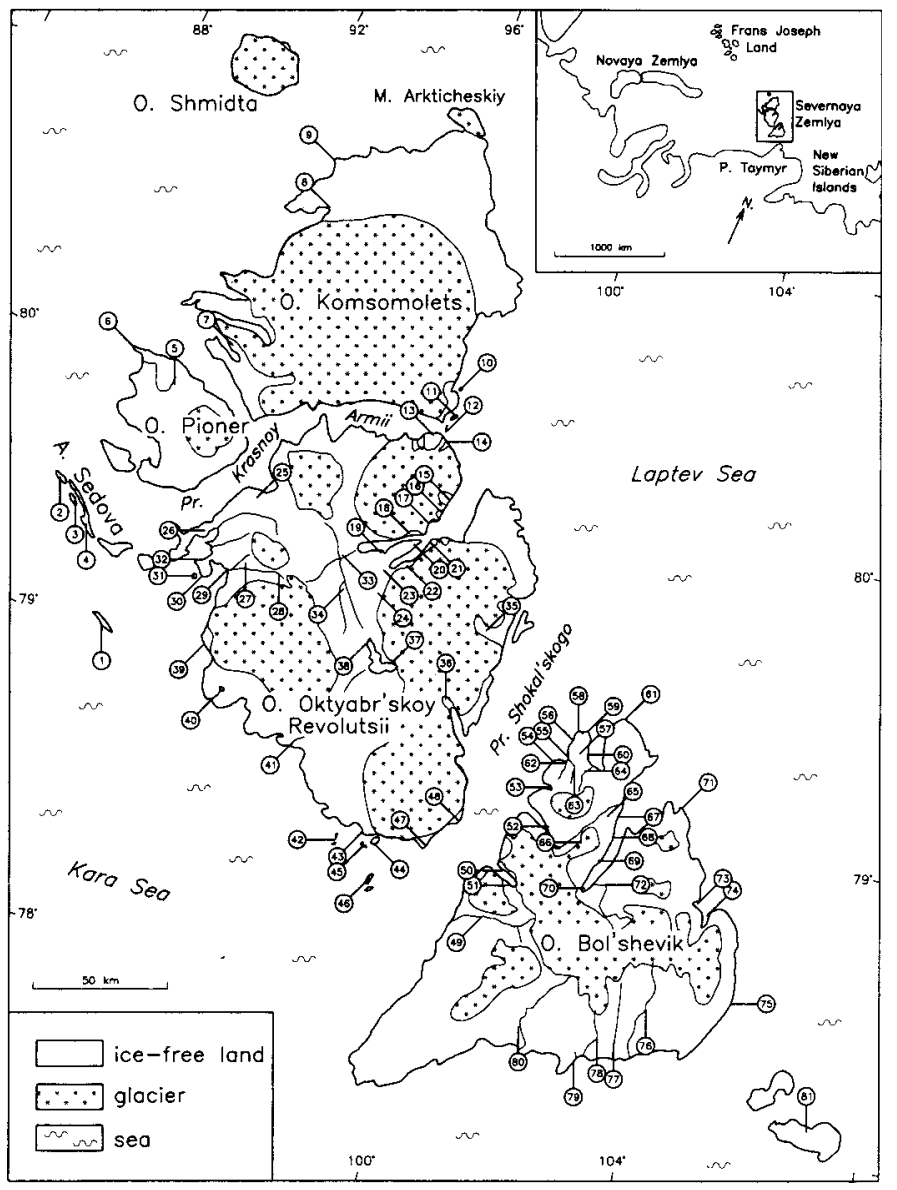

FIG. 1. Map of Severnaya Zemlya with locations of sites listed in Table 1.

\section{GEOGRAPHY AND CLIMATE}

Severnaya Zemlya, with a land area of about $36788 \mathrm{~km}^{2}$, is situated north of Taymyr in Central Siberia, on the boundary of the Kara Sea (Karskoe More) and the Laptev Sea (More 
Laptevykh) between $77^{\circ} 55^{\prime} \mathrm{N}$ and $81^{\circ} 17^{\prime} \mathrm{N}$, and $90^{\circ} 30^{\prime} \mathrm{E}$ and $107^{\circ} 45^{\prime} \mathrm{E}$ (Fig. 1). The four largest islands are O. Bol'shevik (11 $\left.847 \mathrm{~km}^{2}\right)$, O. Oktyabr'skoy Revolutsii $\left(13708 \mathrm{~km}^{2}\right)$, O. Komsomolets $\left(9008 \mathrm{~km}^{2}\right)$, and O. Pioner $\left(1522 \mathrm{~km}^{2}\right)($ Katalog Lednikov, 1980; Dodd, 1985). There are many small islands like O. Sredniy in A. Sedova.

Glaciers cover $49.8 \%$ of the total land area. In the whole archipelago there are wide plains, and mountains covered by dome glaciers, up to a height of $985 \mathrm{~m}$ (O. Bol'shevik). The highest unglaciated mountains reach heights of about $600 \mathrm{~m}$. The north of O. Bol'shevik has the most mountainous area of the whole archipelago. Large areas with flat or undulating tundra occur on all islands. In winter heavy coastal sea ice is formed, which in most areas remains throughout the year. In some areas it disappears for a short period at the end of August. The Eastern Severnaya Zemlya Polynya, along the northern and eastern boundaries of this coastal ice in the Laptev Sea, is close to land and stable (Semenov, 1970). The Western Severnaya Zemlya Polynya, along the western boundaries of fast-ice in the Kara Sea, is unstable and more distant from land.

The climate can be defined as transitional continental to maritime high-arctic. From 1961 until 1991, at the weather station M. Peschannyy on northern O. Bol'shevik, the average temperature of the coldest month (February) was $-29.0^{\circ} \mathrm{C}$, while that of the warmest month (July) was $+0.9^{\circ} \mathrm{C}$. From 1986 until 1991, the average daily minimum for July was $-0.2^{\circ} \mathrm{C}$ and the average daily maximum was $+3.0^{\circ} \mathrm{C}$. Also from 1961 until 1991, average precipitation was $170 \mathrm{~mm}$ per year (N. Adamovitsj, Weather Service Dikson, pers. comm. 1992) and average daily temperatures were above zero from the fourth week of June until the middle of August. At M. Peschannyy, the land was on average $50 \%$ free of snow by 9 July, but at any time during summer the tundra can become completely snow-covered for some days. Common wind speeds in summer are $2-3 \mathrm{~m} \cdot \mathrm{s}^{-1}$, but periods with very strong winds occur regularly. In winter, wind speeds are usually higher. The winds are mostly from the west and southeast.

The northwestern part of the archipelago with $\mathrm{O}$. Komsomolets and $\mathrm{O}$. Pioner is an arctic desert with very sparse vegetation (Semenov, 1967, 1971). In the southeastern part, O. Oktyabr'skoy Revolutsii and O. Bol'shevik (Fig. 1), the vegetation is richer and represented by different types of polygonal communities of herb-lichen-mosses. Locally, in secluded areas which receive moisture during summer, grass hummocks occur (Korotkevich, 1958; Semenov, 1967, 1971; Safronova, 1981, 1993; Khodachek, 1986).

\section{METHODS}

From June until September 1985, Gavrilo (1988a,b) did ornithological studies on O. Oktyabr'skoy Revolutsii between G. Bazarnaya, Oz. F'ordovoe and M. Vatutina, where she had her base. From 18 July until 22 August 1991, Volkov and Pridatko (1994) worked in the northern part of O. Bol'shevik between Z. Akhmatova and Pr. Shokal'skogo, where they had their base in S. Prima. From 15 July until 8
August 1992 and from 13 until 26 July 1993, de Korte and Volkov worked again in this area, making extensive surveys by helicopter of all major islands. From 19 to 23 July 1994, de Korte made a survey by helicopter on O. Bol'shevik, O. Oktyabr'skoy Revolutsii and in A. Sedova. In 1985, 1991 and 1992, we collected the field data mostly during surveys on foot. In 1993 and 1994, we observed mostly from helicopters, flying near localities where, according to previous reports, seabirds were nesting. In most cases we then could confirm the old observation or conclude that the breeding place was deserted. Because the occurrence of terrestrial non-avian predators and prey greatly influences the breeding possibilities of most bird species, a summary of terrestrial mammal observations is also presented in the results.

\section{RESULTS}

Thirty-two bird species have been recorded in the archipelago. Eight species are rather commonly breeding all over the archipelago or in part of it (brent goose, purple sandpiper, glaucous gull, kittiwake, ivory gull, black guillemot, little auk, snow bunting). Nine other species breed in small numbers in very limited areas or infrequently (red-throated diver, king eider, sanderling, Arctic skua, long-tailed skua, herring gull, Arctic tern, snowy owl, Lapland bunting). Two species are uncommon migrant birds (pomarine skua, Ross's gull). The remaining 13 species are rare transient birds.

In Table 1 we have presented only the known or well estimated numbers of breeding pairs of a visited area. Because large parts of the archipelago have not been surveyed, the real numbers for the total archipelago may be much higher. Colonial seabirds dominate in numbers, with a roughly estimated total of between 20000 and 100000 breeding pairs, comprising little auk (10 000-80 000), kittiwake (5000 $-10000)$, black guillemot (1000-5000), ivory gull (1000$2000)$ and glaucous gull (500-1000). Arctic tern (100-500) and herring gull (1-10) have only small populations.

Dominant species of tundra birds all over the archipelago are snow bunting $(1000-10000)$, purple sandpiper (1000$5000)$ and brent goose $(1000-2000)$. The long-tailed skua $(10-100)$ is occasional in lemming areas and is, like the sanderling (100-500), confined to more vegetated areas in O. Oktyabr'skoy Revolutsii and O. Bol'shevik. Arctic skua $(1-10)$ and red-throated diver $(1-10)$ breed in small numbers in O. Bol'shevik, and king eider (1-10), snowy owl (110) and Lapland bunting $(1-10)$ breed in the western part of O. Oktyabr'skoy Revolutsii. Pomarine skua and Ross's gull are uncommon migrants, which in some years are seen in the late summer and autumn. The possibility that a few Ross's gulls sometimes breed in Severnaya Zemlya cannot completely be ruled out (cf. Blomquist and Elander, 1981).

\section{Annotated List of Birds}

Breeding status is defined as follows: breeder-nests or broods recorded; migrant—recorded during migration, but 
TABLE 1. Breeding sites (all bird species), moulting areas (brent goose) and population sizes (order of magnitude) in Severnaya Zemlya. Numbers correspond with numbers in Fig. 1.

\begin{tabular}{|c|c|c|c|c|c|c|c|c|c|c|c|c|c|c|c|c|c|}
\hline \multirow[t]{2}{*}{ Locality $^{1}$} & \multicolumn{17}{|c|}{ Species $^{2}$} \\
\hline & 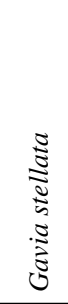 & 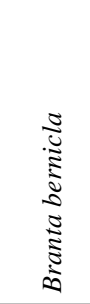 & 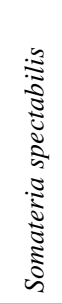 & 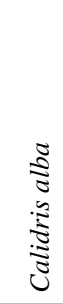 & 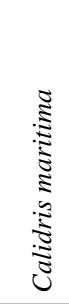 & 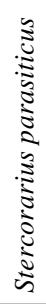 & 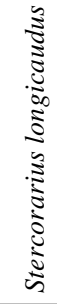 & 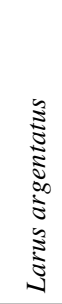 & 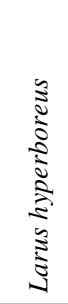 & 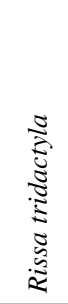 & 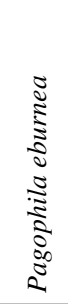 & 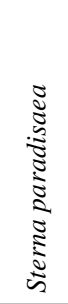 & $\begin{array}{l}\stackrel{\Xi}{\widetilde{\sigma}} \\
\stackrel{\Xi}{\widetilde{Z}}\end{array}$ & 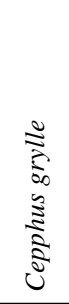 & 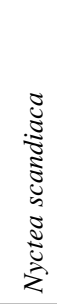 & 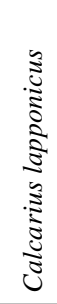 & 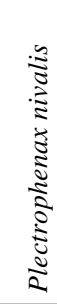 \\
\hline 1. O. Dlinnyy & - & - & - & - & - & - & - & - & - & - & ?b & - & - & - & - & - & - \\
\hline 2. O. Golomyannyy & - & B1 & - & - & - & - & - & - & b1 & $\mathrm{b}$ & b3 & - & - & $\mathrm{b} 2$ & - & - & - \\
\hline 3. O. Domashniy & - & $\mathrm{B} 1$ & - & - & B1 & - & - & - & $\mathrm{B} 1$ & $\mathrm{~b}$ & B4 & - & - & $\mathrm{b} 2$ & - & - & - \\
\hline 4. O. Sredniy & - & - & b1 & - & - & - & - & - & B1 & B4 & b2 & - & - & B2 & - & - & - \\
\hline 5. R. Krugovaya & - & - & - & - & - & - & - & - & - & B2 & - & - & - & - & - & - & - \\
\hline 6. M. Budenogo & - & - & - & - & - & - & - & - & - & - & - & - & - & $\mathrm{b}$ & - & - & - \\
\hline 7. B. Uzlovaya & - & - & - & - & - & - & - & - & B2 & - & - & - & - & - & - & - & - \\
\hline 8. B. Skrytaya & - & - & - & - & - & - & - & - & $\mathrm{B} 1$ & B2 & ?B & - & - & - & - & - & B1 \\
\hline 9. M. Karla Libknekhta & - & - & - & - & - & - & - & - & $\mathrm{b}$ & $\mathrm{b}$ & - & - & $\mathrm{b}$ & $\mathrm{b}$ & - & - & - \\
\hline 10. O. Chistikov & - & - & - & - & - & - & - & - & - & - & - & - & $? \mathrm{~b}$ & B2 & - & - & - \\
\hline 11. O. Machtovyy & - & - & - & - & - & - & - & - & B1 & - & - & - & $? \mathrm{~b}$ & B2 & - & - & - \\
\hline 12. O. Diabazovye & - & - & - & - & - & - & - & - & B1 & - & ?b & - & ?b & B2 & - & - & - \\
\hline 13. M. Goristyy & - & - & - & - & - & - & - & - & - & ?b3 & ?b & - & B4 & B3 & - & - & - \\
\hline 14. M. Voroshilova & - & - & - & - & - & - & - & - & $? \mathrm{~b}$ & - & - & - & $? \mathrm{~b}$ & - & - & - & - \\
\hline 15. F. Matusevicha (north) & - & - & - & - & - & - & - & - & $? \mathrm{~b}$ & - & ?B & - & - & B3 & - & - & - \\
\hline 16. F. Matusevicha (south) & - & - & - & - & - & - & - & - & ?b & - & - & - & B & B3 & - & - & - \\
\hline 17. G. Mira & - & - & - & - & - & - & - & - & - & - & - & - & $\mathrm{B}$ & - & - & - & - \\
\hline 18. B. Krasnaya (north) & - & - & - & - & - & - & - & - & B1 & B3 & - & - & ?B & B3 & - & - & - \\
\hline 19. B. Krasnaya (south) & - & - & - & - & - & - & - & - & B1 & - & - & - & ?B & B3 & - & - & - \\
\hline 20. G. Bazarnaya & - & - & - & - & - & - & - & - & $\mathrm{B} 2$ & B3 & ?b & - & B4 & B3 & - & - & - \\
\hline 21. B. Skazochnaya (north) & - & - & - & - & - & - & - & - & - & - & - & - & B4 & B & - & - & - \\
\hline 22. B. Skazochnaya (middle) & - & - & - & - & - & - & - & - & - & - & - & - & B4 & $\mathrm{B}$ & - & - & - \\
\hline 23. B. Skazochnaya (south) & - & - & - & - & - & - & - & - & B1 & - & B1 & - & - & B3 & - & - & - \\
\hline 24. R. Lednikovaya & - & - & - & - & - & - & - & - & $\mathrm{B} 1$ & - & - & - & - & - & - & - & - \\
\hline 25. M. Serp i Molot & - & - & - & - & - & - & - & - & - & - & - & - & - & - & b1 & - & - \\
\hline 26. P. Parizhskoy Kommuny & - & - & - & - & B1 & - & - & - & - & - & ?b & B1 & - & - & - & B1 & B2 \\
\hline 27. R. Gremyashchaya & - & - & B1 & - & B1 & - & B1 & - & B2 & - & - & - & - & - & - & B1 & B2 \\
\hline 28. R. Pod'emnaya & - & $\mathrm{M}$ & - & - & B1 & - & B1 & - & - & - & - & - & - & - & - & - & B2 \\
\hline 29. Z. Panfilovtsev & - & M & - & - & B1 & - & - & - & - & - & - & - & - & - & - & - & $\mathrm{B} 2$ \\
\hline 30. M. Vatutina & - & - & - & B1 & B1 & - & - & - & - & - & - & - & - & - & - & - & - \\
\hline 31. O. Obmannyy & - & - & - & $\mathrm{B} 1$ & B1 & - & - & - & - & - & - & - & - & - & - & - & - \\
\hline 32. P. Zhiloy & - & B1 & B1 & - & B1 & - & - & - & - & - & - & - & - & - & - & - & B2 \\
\hline 33. R. Matusevicha & - & - & - & - & - & - & - & - & B2 & - & - & - & - & - & b1 & - & - \\
\hline 34. R. Ushakova & - & - & - & - & - & - & - & - & B1 & - & - & - & - & - & - & - & - \\
\hline 35. G. Tumannye & - & - & - & - & - & - & - & - & B2 & - & - & - & - & - & - & - & - \\
\hline 36. G. Ploskaya (north) & - & - & - & - & - & - & - & - & - & - & B2 & - & - & - & - & - & - \\
\hline 37. Oz. F'ordovoe (east) & - & - & - & - & - & - & - & - & B1 & B4 & - & - & - & - & - & - & - \\
\hline 38. Oz. F'ordovoe (north) & - & - & - & - & - & - & - & - & B1 & - & - & - & - & - & - & - & - \\
\hline 39. M. Krzhizhanovskogo & - & $\mathrm{m}$ & - & - & - & - & - & - & - & - & - & - & - & - & - & - & - \\
\hline 40. O. Izmenchivoe (river) & - & b2M2 & - & - & - & - & - & - & B2 & B3 & ?B & - & - & B2 & - & - & - \\
\hline 41. R. Ozernaya & - & - & - & - & - & - & - & - & $\mathrm{B}$ & B3 & - & - & - & $\mathrm{b} 2$ & - & - & - \\
\hline 42. O. Olen'i & - & - & - & - & - & - & - & - & B1 & B2 & - & - & - & - & - & - & - \\
\hline 43. M. Sverdlova & - & $\mathrm{m}$ & - & - & - & - & - & - & - & $\mathrm{B} 2$ & - & - & - & - & - & - & - \\
\hline 44. O. Sverdlova & - & - & - & - & - & - & - & - & B1 & B2 & - & - & - & - & - & - & - \\
\hline 45. islets off O. Sverdlova & - & - & - & - & - & - & - & - & B1 & B2 & - & - & - & - & - & - & - \\
\hline 46. O. Bol'shoy & - & - & - & - & - & - & - & - & $\mathrm{b}$ & - & $\mathrm{b}$ & - & - & - & - & - & $\mathrm{b}$ \\
\hline 47. M. Massivnyy & - & - & - & - & - & - & - & - & - & - & ?b & - & - & - & - & - & - \\
\hline 48. M. Olovyannyy & - & - & - & - & - & - & - & - & $\mathrm{B}$ & B2 & ?b & - & $? \mathrm{~b}$ & B2 & - & - & - \\
\hline 49. R. Studenaya & - & BM2 & - & - & - & - & - & - & B2 & B2 & ?B & - & - & - & - & - & - \\
\hline 50. F. Tel'mana (north) & - & - & - & - & - & - & - & - & B2 & - & - & - & $? \mathrm{~b}$ & B2 & - & - & - \\
\hline 51. F. Tel'mana (south) & - & - & - & - & - & - & - & - & B2 & - & - & - & $? \mathrm{~b}$ & B2 & - & - & - \\
\hline 52. F. Spartak & - & - & - & - & - & - & - & - & - & ?B & - & - & - & $\mathrm{B} 2$ & - & - & - \\
\hline 53. F. Partizanskiy & - & - & - & - & - & - & - & - & B1 & B3 & - & - & - & B2 & - & - & - \\
\hline 54. M. Vize & - & - & - & - & - & - & - & - & B1 & - & - & - & - & B2 & - & - & - \\
\hline 55. B. Amba & - & B1 & $\mathrm{B} 1$ & - & B1 & - & - & - & - & - & - & $\mathrm{B} 1$ & - & - & - & - & - \\
\hline 56. S. Prima & - & - & - & - & B1 & - & - & - & - & - & - & - & - & - & - & - & - \\
\hline 57. Oz. Tverdoe & ?B1 & - & - & - & B1 & - & - & - & - & - & $\mathrm{B} 1$ & - & - & - & - & - & - \\
\hline 58. M. Zub & - & B1 & - & - & B1 & - & - & B1 & - & - & - & B1 & - & - & - & - & - \\
\hline 59. M. Baranova & - & $\mathrm{B} 1$ & - & - & B1 & - & - & - & - & - & - & - & - & - & - & - & $\mathrm{B} 2$ \\
\hline 60. Z. Mikoyana & - & B1 & - & B1 & B1 & - & - & - & - & - & - & - & - & - & - & - & - \\
\hline
\end{tabular}




\begin{tabular}{|c|c|c|c|c|c|c|c|c|c|c|c|c|c|c|c|c|c|}
\hline \multirow[t]{2}{*}{ Locality $^{1}$} & \multicolumn{17}{|c|}{ Species $^{2}$} \\
\hline & 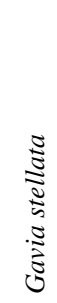 & 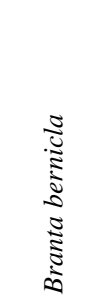 & 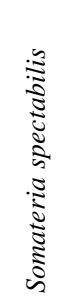 & 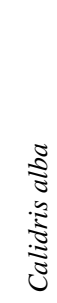 & 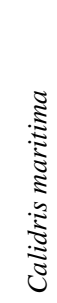 & 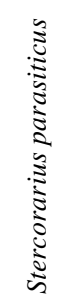 & 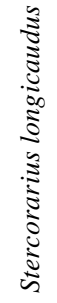 & 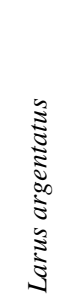 & 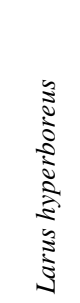 & 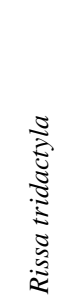 & 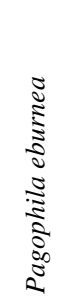 & 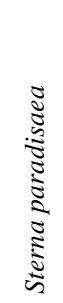 & $\begin{array}{l}\stackrel{\Xi}{\Xi} \\
\stackrel{\Xi}{\widetilde{\pi}}\end{array}$ & 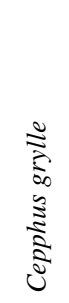 & 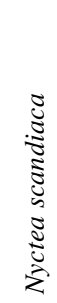 & 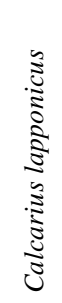 & 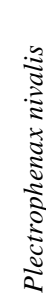 \\
\hline 61. M. Peschannyy & - & - & - & - & B1 & - & - & - & - & - & ?B & - & - & - & - & - & - \\
\hline 62. R. Amba & - & $\mathrm{B} 1 \mathrm{M} 2$ & - & - & B1 & - & - & - & ?B1 & - & - & - & - & - & - & - & - \\
\hline 63. R. Mushketova & - & - & - & - & - & - & - & - & - & - & ?B & - & - & - & - & - & - \\
\hline 64. R. Ostantosvaya & - & - & - & - & - & B1 & - & - & B1 & B2 & B2 & - & - & B2 & - & - & B2 \\
\hline 65. R. Bazovaya & B1 & B2 & - & - & B1 & B1 & B1 & - & ?B1 & - & - & - & - & B2 & - & - & B2 \\
\hline 66. L. Voytsekhovskogo & - & - & - & - & - & - & - & - & - & - & B2 & - & - & - & - & - & - \\
\hline 67. Z. Akhmatova (north) & - & M3 & - & B1 & B1 & - & B1 & B1 & - & - & - & - & - & - & - & - & B2 \\
\hline 68. G. Zvezdina & - & - & - & - & - & B1 & - & ?B & ?B1 & - & - & - & - & ?B & - & - & - \\
\hline 69. Z. Akhmatova (middle) & - & - & - & - & - & - & - & - & - & B3 & - & - & - & - & - & - & - \\
\hline 70. Z. Akhmatova (south) & - & BM3 & - & - & - & - & - & - & - & - & - & - & - & - & - & - & - \\
\hline 71. M. Tyazhelyy & - & M2 & - & - & - & - & - & - & - & - & - & - & - & - & - & - & - \\
\hline 72. R. Slozhnaya & B1 & B1 & - & - & - & - & - & - & B1 & - & - & - & - & - & - & - & - \\
\hline 73. B. Tumannaya (north) & & M2 & & & & & - & - & - & - & - & - & - & - & - & - & - \\
\hline 74. B. Tumannaya (south) & - & BM2 & - & - & - & - & - & - & - & - & - & - & - & - & - & - & - \\
\hline 75. M. Morozova & - & - & - & - & - & - & - & - & B1 & - & - & - & - & - & - & - & - \\
\hline 76. R. Skalistaya & - & - & - & - & - & - & - & - & ?B1 & B3 & - & - & - & - & - & - & - \\
\hline 77. R. Lagernaya & - & - & - & - & - & - & B1 & - & B1 & - & - & - & - & - & - & - & - \\
\hline 78. R. Shumnaya & - & BM2 & - & - & - & - & - & - & ?B1 & - & $\mathrm{B} 2$ & - & - & - & - & - & - \\
\hline 79. B. Solnechnaya & - & - & - & - & B1 & - & - & - & - & - & - & B1 & - & - & - & - & $\mathrm{B} 2$ \\
\hline 80. R. Tora & - & - & - & - & - & - & - & - & ?B1 & ?B3 & - & - & - & - & - & - & - \\
\hline 81. O. Malyy Taymyr & - & B & - & - & - & - & - & - & - & - & ?B & - & - & - & - & - & - \\
\hline All Severnaya Zemlya & B1 & B3M4 & $\mathrm{B} 1$ & B2 & B3 & B1 & B2 & B1 & B3 & B4 & B4 & $\mathrm{B} 2$ & B5 & B4 & b1 & $\mathrm{B} 1$ & B4 \\
\hline
\end{tabular}

${ }^{1} \mathrm{~B}=$ Bukhta $=$ Bay, $\mathrm{F}=\mathrm{F}$ 'ord = Fjord, $\mathrm{G}=$ Gora $=$ Mountain, $\mathrm{L}=$ Lednik $=$ Glacier, $\mathrm{M}=\mathrm{Mys}=\mathrm{Cape}, \mathrm{O}=\mathrm{Ostrov}=\mathrm{Island}, \mathrm{Oz}=\mathrm{Ozero}$ $=$ Lake, $\mathrm{P}=$ Poluostrov = Peninsula, $\mathrm{R}=$ Reka $=$ River, $\mathrm{S}=$ Stantsiya $=$ Station, $\mathrm{Z}=$ Zaliv = Gulf.

${ }^{2} \mathrm{~b}=$ breeding, (?) $\mathrm{b}=$ (probable) breeding recorded before 1980, (?)B = (probable) breeding recorded in and after 1980; $\mathrm{B} 1$ and $\mathrm{b} 1=$ 1 - 9 breeding pairs, B2 and b2 $=10-99$ breeding pairs, B3 and b3 = 100-999 breeding pairs, B4 and b4 = 1000-9999 breeding pairs, B5 = 10 000-99 999 breeding pairs; $\mathrm{m}=$ moulting area recorded before 1980, $\mathrm{M}=$ moulting area recorded in and after $1980, \mathrm{M} 2=10-$ 99 moulting individuals, M3 = 100-999 moulting individuals, M4 = 1000-9999 moulting individuals.

no evidence of breeding; transient—non-breeding bird or irregular visitors outside of their known geographic distribution. Categories of relative abundance of breeding species are defined by the estimates of the total numbers of breeding pairs in the whole archipelago: rare $(<10)$; occasional $(10-99)$; uncommon (100-999); common (1000-9999); abundant (> 10000$)$.

Red-throated Diver (Gavia stellata): Rare breeder. Single birds and pairs were observed on A. Sedova (Belikov and Randla, 1987), near M. Vatutina in 1985, and near Oz. Tverdoe in 1992 and 1993, where an old nest was also found. Volkov and Pridatko (1994) saw a pair on a lake near R. Bazovaya on 2 August 1991. At this lake we saw a nest with two eggs on 20 July 1992, but only a pair on 16 July 1993. A pair with large young was seen on 25 August 1982 in R. Slozhnaya (Bulavintsev, 1984). The earliest recorded arrival, on 29 June 1948, is from O. Domashniy (unpubl. logbook, Polar Station, O. Domashniy).

Fulmar (Fulmarus glacialis): Rare transient. Fulmars have been reported at sea along the western coasts of the archipelago (Laktionov, 1936). A few single birds and flocks were seen in August and September in A. Sedova and, in July, southeast of
O. Oktyabr'skoy Revolutsii (Urvantsev, 1935; Ushakov, 1951).

Brent Goose (Branta bernicla): Uncommon breeder. Brent geese breed in A. Sedova (Demme, 1934), where four nests were found on O. Golomyannyy (Kharitonovich, 1940). On 15 July 1993, we found three nests on O. Domashniy, containing three, three and five eggs respectively; on 22 July two of these nests were still occupied by incubating birds; the third nest and a fourth, found on that day, were empty. On this island we saw two breeding pairs on 20 July 1994. On 22 July 1985, two empty nests were found on P. Zhiloy. Brent geese moult near Z. Panfilovtsev and in the valley of R. Pod'emnaya as noted by Laktionov, (1936) and by us in 1985. Moulting groups have been observed along the south of Pr. Krasnoy Armii and at M. Krzhizhanovskogo (Urvantsev, 1935). On an islet in Oz. Izmenchivoe, three pairs bred in 1976 and, near the eastern bank, 10 pairs in 1979 (V.M. Makeev, pers. comm. 1986). Groups of up to 100 flightless birds were seen here in 1975 and up to 40 in 1983 (V.M. Makeev, pers. comm. 1986; Belikov and Randla, 1987). Moulting groups are also reported from M. Sverdlova (Ushakov, 1951). Flightless adults and young were observed in 1982 by Bulavintsev (1984) at the mouth of R. Studenaya (about 50), near the head 
of Z. Akhmatova (about 200), in B. Tumannaya (about 50), in R. Shumnaya (about 50) and, on 27 August, in R. Slozhnaya ( 2 families, 8 young).

Near S. Prima one nest was found in 1987 (V.V. Baranov, pers. comm. 1992). From 1 to 21 August 1991 Volkov and Pridatko (1994) saw a total of 27 flightless families with on average 4.5 young between Z. Akhmatova and R. Bazovaya and six families near S. Prima. On 17 July 1992 about $50 \%$ of about 260 moulting adults in Z. Akhmatova were flightless, on 21 July about $95 \%$, and on 24 July all of them, but on 30 July one single bird was seen flying along the west coast of $Z$. Mikoyana. In 1993 we saw on 14 July two breeding pairs near S. Prima, on 16 July about 40 flightless adults near M. Tyazhelyy and 20 in B. Tumannaya, on 17 July six breeding pairs, a family and an empty nest along the western side of $Z$. Mikoyana, and on 26 July a family with five young and a pair without young south of M. Zub. On 20 July 1994 we found a nest with four eggs near Z. Amba. On O. Malyy Taymyr several pairs bred in 1993 (personnel, Polar Station, O. Malyy Taymyr, pers. comm. 1993).

The earliest recorded arrival dates vary from 12 to 17 June in A. Sedova and Pr. Shokal'skogo, when the brent geese were seen in flocks of up to 20 birds; within six days after arrival the females had complete eggs in the ovaries (Demme, 1934; Urvantsev, 1935; Ushakov, 1951; unpubl. logbooks, Polar Station, 1931, 1932, 1933, 1934, 1936, 1952). In 1992 brent geese were mostly seen in groups until 24 June, but after 1 July mostly in separate pairs (C. Niemitz, pers. comm. 1993). The observation latest in the year is from 12 September, when flocks of about 10 and 40 were seen at the southern tip of P. Parizhskoy Kommuny (A. Ryuss, pers. comm. 1985).

Long-tailed Duck (Clangula hyemalis): Rare transient. In 1931 a few groups were seen at O. Golomyannyy (Demme, 1934). On 14 July 1950, a group of 10 was observed on the southern coast of O. Oktyabr'skoy Revolutsii (E.S. Korotkevich, pers. comm. 1986). One was seen on P. Parizhskoy Kommuny (Semenov, 1967). This bird may breed on O. Bol'shevik (Belikov and Randla, 1987).

Common Eider (Somateria mollissima): Rare transient. Two males and a female were seen in July 1992 in the north of O. Komsomolets (C. Niemitz, pers. comm. 1993).

King Eider (Somateria spectabilis): Rare breeder. On 31 July 1930, a family with 11 ducklings was seen on O. Sredniy (Demme, 1934). On 4 July 1932, a male was shot in A. Sedova (Urvantsev, 1935). At the end of July 1983, Belikov and Randla (1987) saw families with three and five recently hatched young on P. Zhiloy. Near Z. Panfilovtsev, pairs and mixed groups of up to 14 birds were observed by Gavrilo (1988b) from 2 to 27 July 1985. On 10 July, she found near R. Gremyashchaya a nest with five eggs, the young of which had hatched by 16 July. On 19 July and 13 August 1991, Volkov and Pridatko (1994) saw groups of two and three females respectively in a lagoon at B. Amba, where on $20 \mathrm{July}$ 1994 again a female was seen. Along the western shore of $Z$. Mikoyana, we saw a female on 22 July 1994. At the coast of eastern O. Malyy Taymyr we saw a group of one male and two females on 16 July 1993. It bred in 1995 at B. Amba.
The earliest recorded appearance of this bird is on 27 May 1950 on O. Domashniy (E.S. Korotkevich, pers. comm. 1986).

Peregrine Falcon (Falco peregrinus): Rare transient. This species has been observed on O. Bol'shoy (unpubl. logbook, Polar Station, O. Bol'shoy, 1956).

Rock Ptarmigan (Lagopus mutus): Rare transient. This species possibly occurs in O. Oktyabr'skoy Revolutsii, where excrements were found near B. Krasnaya (north) in 1985, and in O. Bol'shevik (Belikov and Randla, 1987; Y. Kotlov, pers. comm. 1992).

Knot (Calidris canutus): Rare transient. This species was seen in A. Sedova by Semenov (1971). E.S. Korotkevich (pers. comm. 1986) saw a few birds on four occasions from 22 July to 14 August 1950 in the central parts of $O$. Oktyabr'skoy Revolutsii. On 3 July 1985 Gavrilo (1988a) saw one foraging along the coast at M. Vatutina.

Sanderling (Calidris alba): Occasional breeder. This species has been observed on western O. Oktyabr'skoy Revolutsii (Laktionov, 1936). Belikov and Randla (1987) saw small groups on P. Zhiloy and P. Parizhskoy Kommuny on 4 and 5 August 1983. Gavrilo (1988a) found one nest at M. Vatutina on 22 July 1985, one at P. Parizhskoy Kommuny on 27 July, and the first brood of that year on O. Obmannyy on 20 July. On 21 July 1992 we found a nest with four eggs near Z. Akhmatova, and on 30 July a family with one young of about five days age near Z. Mikoyana.

Purple Sandpiper (Calidris maritima): Uncommon breeder. On O. Domashniy we saw a bird exhibiting distraction behaviour on 22 July 1993, and on 20 July 1994 four foraging adults. A foraging adult was seen in July 1993 in B. Skrytaya (C. Niemitz, pers. comm. 1993). Gavrilo (1988a) counted in the western part of O. Oktyabr'skoy Revolutsii between Z. Panfilovets and Pr. Krasnoy Armii in July 1985 about 1.6 bird per $\mathrm{km}$ on the coastal plains and about 0.4 bird per $\mathrm{km}$ between the rivers more inland. She found a total of 24 broods from 16 to 25 July, including seven on O. Obmannyy $\left(2.2 \mathrm{~km}^{2}\right)$ on 23 July. Demme (1934) reports breeding at M. Krzhizhanovskogo. Between Pr. Shokal'skogo and Z. Akhmatova, Volkov and Pridatko (1994) counted about 0.4 birds per $\mathrm{km}$ in 1991. They found two families near S. Prima on 18 and 12 August and saw an egg from early July at M. Peschannyy. In 1992 we counted also about 0.4 birds per km and found two nests and six broods in the same area (Fig. 2). These broods were estimated to have hatched between 17 and 28 July, with an average hatching date of 22 July. On 14 July and 18 July 1993, we found two nests with four eggs near B. Amba, which had hatched by 24 and 26 July respectively. Near B. Solnechnaya, Bulavintsev (1984) found a clutch on 20 July 1982.

The earliest recorded arrival dates are 14 June 1952 and 16 June 1951 on O. Domashniy (unpubl. logbook, Polar Station).

Sharp-tailed Sandpiper (Calidris acuminata): Rare transient. On 4 July 1985 Gavrilo (1988a) saw one pair of this species $0.5 \mathrm{~km}$ inland near Z. Panfilovtsev.

Red Phalarope (Phalaropus fulicarius): Rare transient. This species was recorded flying in A. Sedova (Semenov, 1971).

Great Skua (Catharacta skua): Rare transient. On 23 July 


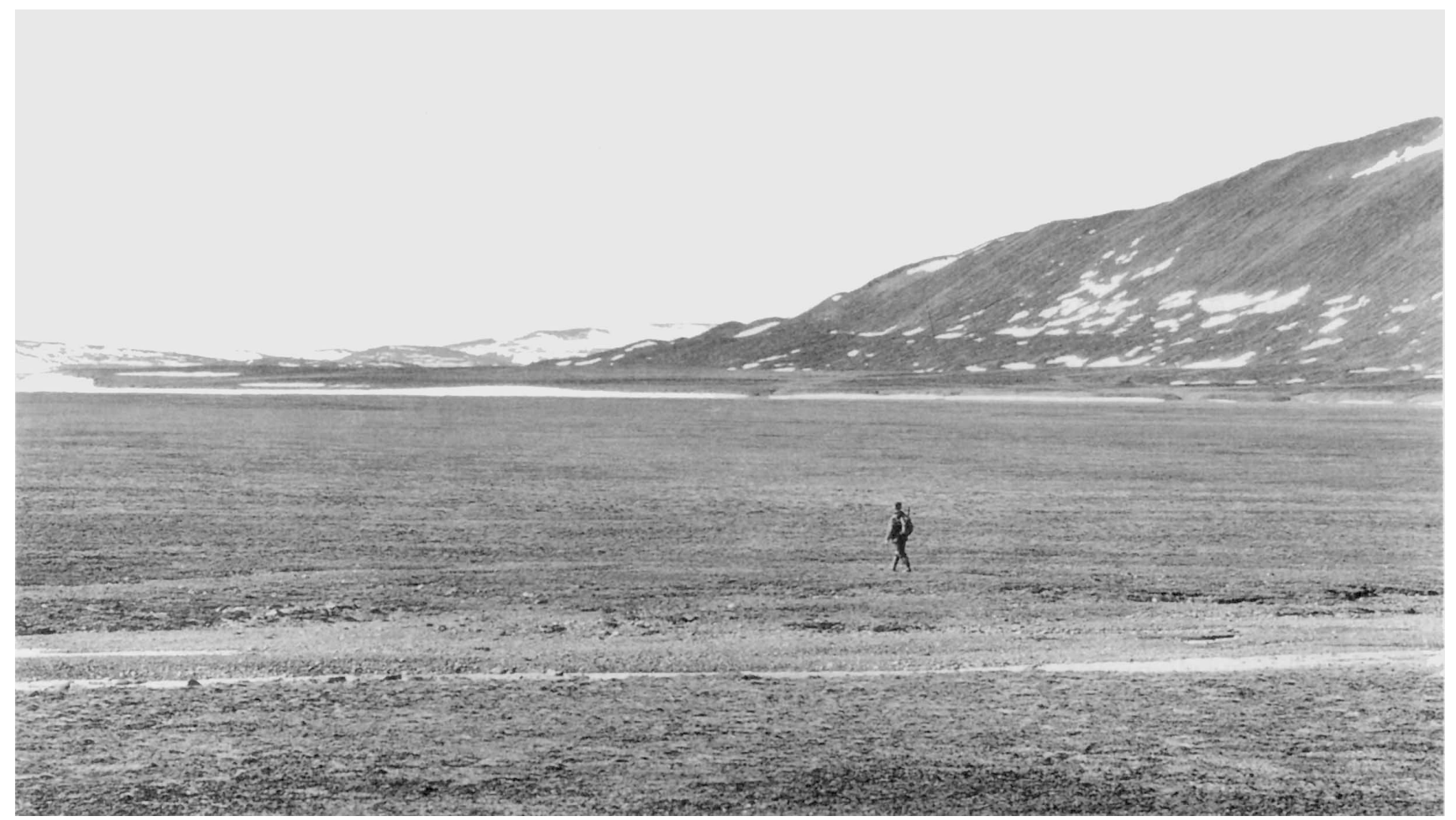

FIG. 2. Habitat of purple sandpiper and long-tailed skua near Z. Akhmatova on 20 July 1992. (Photo: J. de Korte).

and 2 August 1991 Volkov and Pridatko (1994) saw on both dates two birds near S. Prima.

Pomarine Skua (Stercorarius pomarinus): Uncommon migrant. In July 1985, flocks of up to 50 birds were seen near P. Zhiloy and a few birds near G. Bazarnaya and inland in O. Oktyabr'skoy Revolutsii. Along the coast of B. Amba, Volkov and Pridatko (1994) observed groups of up to four birds between 27 July and 18 August 1991. In B. Amba about a dozen were chasing kittiwakes and ivory gulls on 26 and 29 July 1992. On 1 August, we saw a group of 13 on the sea ice near M. Baranova. On the ice along the southeast coast of O. Malyy Taymyr, we observed small groups totalling about 150 birds on 16 July 1993. On 22 July 1994 we saw four birds near S. Prima.

Arctic Skua (Stercorarius parasiticus): Rare breeder. The Arctic skua has been seen in A. Sedova (Semenov, 1971), near P. Zhiloy and in central O. Oktyabr'skoy Revolutsii (1985), Oz. F'ordovoe (Belikov and Randla, 1987), and northern O. Bol'shevik (Bulavintsev, 1984). Near a lake between R. Bazovaya and Z. Akhmatova, Volkov and Pridatko (1994) encountered a bird exhibiting distraction behaviour on 2 August 1991. We found one nest with two eggs on 19 July 1992, near R. Bazovaya; one nest on 22 July along the coast near G. Zvezdina; and one on 30 July, near a lake about $3 \mathrm{~km}$ west of R. Ostantsovaya. On 17 July 1993, we saw the pair of the latter nest in the same area again, with a nest containing two eggs. On 22 July 1994 there was only one bird in this territory.

Long-tailed Skua (Stercorarius longicaudus): Occasional breeder. On 26 July 1985, on the low tundra near R. Pod'- emnaya, we met on average one territorial pair per $17 \mathrm{~km}$, among which were a pair with distraction behaviour, and a pair with a chick. Between R. Bazovaya and Z. Akhmatova, Volkov and Pridatko (1994) met three attacking pairs on 3 August 1991. We found six territorial pairs in the same area in 1992, one of which had two eggs on 18 July and another with one egg on 23 July. In 1993 we saw only a few nonbreeding pairs here. We saw a few single adults on the tundra near R. Mushketova before 30 July 1992. On 4 August we saw a group of nine birds, including three immatures, flying across the tundra near R. Ostantsovaya. In the valley of R. Lagernaya, Bulavintsev (1984) found one nest with two eggs on 20 July 1982.

The earliest recorded observation date is 3 June 1948 at O. Domashniy (unpubl. logbook, Polar Station, O. Domashniy).

Herring Gull (Larus argentatus): Rare breeder. Specimens were collected in the eastern part of the archipelago in 1913 and 1950 (Nasonov, 1916; Semenov, 1967; E.S. Korotkevich, pers. comm. 1986). Laktionov (1936) reports one bird in A. Sedova. On 7 August 1992, we saw an immature on O. Golomyannyy. In July 1991, Volkov and Pridatko (1994) saw four adults and an immature on the sea-ice in B. Amba. On 15 and 27 July 1992, we observed three alarm-calling pairs and three empty nests at M. Zub. On 17 July we found one nest with one egg and an empty nest with an alarm-calling pair in the delta of a small river in the north of Z. Akhmatova. We saw three more alarmcalling pairs near G. Zvezdina on 22 July. On 26 July 1993, we saw three alarm-calling pairs and found a nest with one 
egg at M. Zub. On O. Malyy Taymyr we saw about 20 birds on 16 July 1993.

Glaucous Gull (Larus hyperboreus): Uncommon breeder. This bird breeds on O. Golomyannyy (Laktionov, 1936), and on O. Sredniy (D.J. Bolshiyanov, pers. comm. 1990). On 15 July 1993, about $90 \%$ of the young of seven pairs on O. Domashniy had hatched, but on 20 July 1994, none of four nests had chicks. Hundreds of glaucous gulls breed at B. Uzlovaya (Belikov and Randla, 1987) and a few pairs breed at B. Skrytaya (A. Yunak, pers. comm. 1992). Laktionov (1936) reports breeding glaucous gulls at R. Shar'yazhnaya (O. Komsomolets) and M. Karla Libknekhta. In 1993 we saw some breeding pairs at B. Skrytaya and on O. Diabazovye and several pairs on O. Machtovyy.

Glaucous gull colonies occur on G. Bazarnaya, mainly on the north side, with some dozens of breeding pairs (Belikov and Randla, 1987). These colonies were seen by us in 1985, 1993 and 1994, and some pairs were seen on the north side of B. Krasnaya in 1993 and 1994. The reports of breeding in F. Matusevicha and on M. Voroshilova by Laktionov (1936) were not confirmed in 1993. In 1985 Gavrilo (1988b) found single nests south of B. Krasnaya and south of B. Skazochnaya, two nests in the valley of R. Ushakova, 16 nests along R. Matusevicha and 14 nests near R. Gremyashchaya. On 10 July, about $60 \%$ of the young from these 14 nests (mean clutch size 2.9) had hatched, while the largest young were about six days old. In R. Lednikovaya, we saw a pair on 23 July 1994. On G. Tumannye, about $30 \mathrm{~km}$ from the coast, a colony of about 20 pairs was reported by Belikov and Randla (1987). At Oz. F'ordovoe, we saw one pair at the north side of the lake with two young of about three weeks old on 7 August 1992. Glaucous gulls were also seen on the east side of a large kittiwake colony in 1992, 1993 and 1994. There are colonies of some tens of pairs in the canyons of the river from Oz. Izmenchivoe and of R. Ozernaya (Belikov and Randla, 1987; E. Korotkevich, pers. comm. 1988; our observations, 1992, 1993). We saw in 1993 and 1994 colonies of less than 10 pairs on the northernmost island of O. Olen'i and on two islets just southwest of O. Sverdlova. Glaucous gulls bred on O. Bol'shoy in 1956 (unpubl. logbook, Polar Station, O. Bol'shoy). On M. Olovyannyy a few breeding pairs were noted by Urvantsev (1935) and Ushakov (1951) and again by us in 1994.

In the canyon of R. Studenaya and on both sides of F. Tel'mana we saw in 1992, 1993 and 1994 some dozens of pairs. Nine breeding pairs were found at F. Partizanskiy and two south of M. Vise in 1991, one of which was with two young of about 10 days old on 24 July (Volkov and Pridatko, 1994). In 1992 we found a breeding pair and two alarmcalling pairs in R. Ostantsovaya. On 16 July the former pair was still incubating, but by 25 July the oldest of their three young was about one week old. We saw also an alarm-calling pair near G. Zvezdina, R. Bazovaya and R. Amba. We saw a small colony near M. Morozova in 1993 and single pairs in R. Slozhnaya and R. Lagernaya in 1994. Bulavintsev (1984) reports glaucous gulls in the south of O. Bol'shevik, with some pairs nesting along each of the larger rivers.

The earliest recorded arrival date is 8 April at G. Bazarnaya in 1931; on 15 May there were many in a polynya north of M. Arkticheskiy (Ushakov, 1951). On 14 September 1983, Belikov and Randla (1987) saw a just-fledged juvenile at G. Bazarnaya. At O. Domashniy, glaucous gulls arrived in early May and left at the end of October in 1934 (Demme, 1934).

Sabine's Gull (Xema sabini): Rare transient. Single observations are reported from O. Oktyabr'skoy Revolutsii (Semenov 1971). The wings of a Sabine's gull were found on 12 July 1950 on the south coast of O. Oktyabr'skoy Revolutsii (Sdobnikov, 1960).

Ross's Gull (Rhodostethia rosea): Uncommon migrant. In September 1931, 20 to 30 birds passed O. Domashniy (Urvantsev, 1935; Ushakov, 1951). At the end of the summer of 1976, some hundreds were foraging on the northern shore of O. Komsomolets (V.M. Makeev, pers. comm. 1986; Belikov and Randla, 1987). In July 1991, 1992 and 1993, some adults and immatures were foraging in open leads among the sea-ice between M. Vize and M. Zub and in Z. Mikoyan. Single birds and small groups occur every year in B. Solnechnaya (Belikov and Randla, 1987).

Kittiwake (Rissa tridactyla): Common breeder. On O. Golomyannyy and O. Domashniy breeding has been reported by Demme (1934). On O. Domashniy, clutches were completed in the first decade of July 1933 and the first flying young of the year was seen on 16 August (Demme, 1934). On 22 July 1993 and 20 July 1994, we found here a clutch of two eggs in the middle of a large ivory gull colony on flat ground. On the south coast of O. Sredniy there are colonies with a total of about 1000 pairs (D. Bol'shiyanov, pers. comm. 1990; our observations, 1992, 1993, 1994). The nests contained mainly one egg and no young on 14 July 1993 and 22 July 1994. In the canyon of R. Krugovaya we saw two colonies of some dozens of pairs in 1993. Near B. Skrytaya about 100 pairs breed in sandstone columns (A. Yunak, per. comm. 1992). According to Laktionov (1936) they breed on M. Karla Libknekhta. A large colony reported by Semenov (1971) on M. Goristyy was not seen by us in 1993, but on the north side of B. Krasnaya and G. Bazarnaya we found colonies of more than 100 pairs. Along the northeast coast of Oz. F'ordovoe, there was a colony of about 4000-5000 birds with still many flightless young on 12 September 1983 (Belikov and Randla, 1987), and about 2300 pairs with no young yet on 14 July 1993 and 20 July 1994. Along the west side of the river from Oz. Izmenchivoe, there is a colony of about 500 pairs (Belikov and Randla, 1987; our observations, 1992, 1993). Many flightless young were still present here on 8 September 1983 (Belikov and Randla, 1987). In the canyon of R. Ozernaya near the coast, we noted in 1993 a colony of about 500 pairs. In this colony on 11 July 1950 clutches had one or two eggs (E.S. Korotkevich, pers. comm. 1988). On the southern and northern islands of O. Olen'i, on M. Sverdlova, on O. Sverdlova and on two islets south of O. Sverdlova we saw in 1993 and 1994 colonies of some dozens of pairs, and at M. Olovyannyy we counted about 250 pairs. Bulavintsev (1984) reports a colony at R. Studenaya, where we saw a few pairs in 1994 . On 26 July 1991, Volkov and Pridatko (1994) saw a gull colony at the head of F. Spartak and a colony of 400 pairs of 


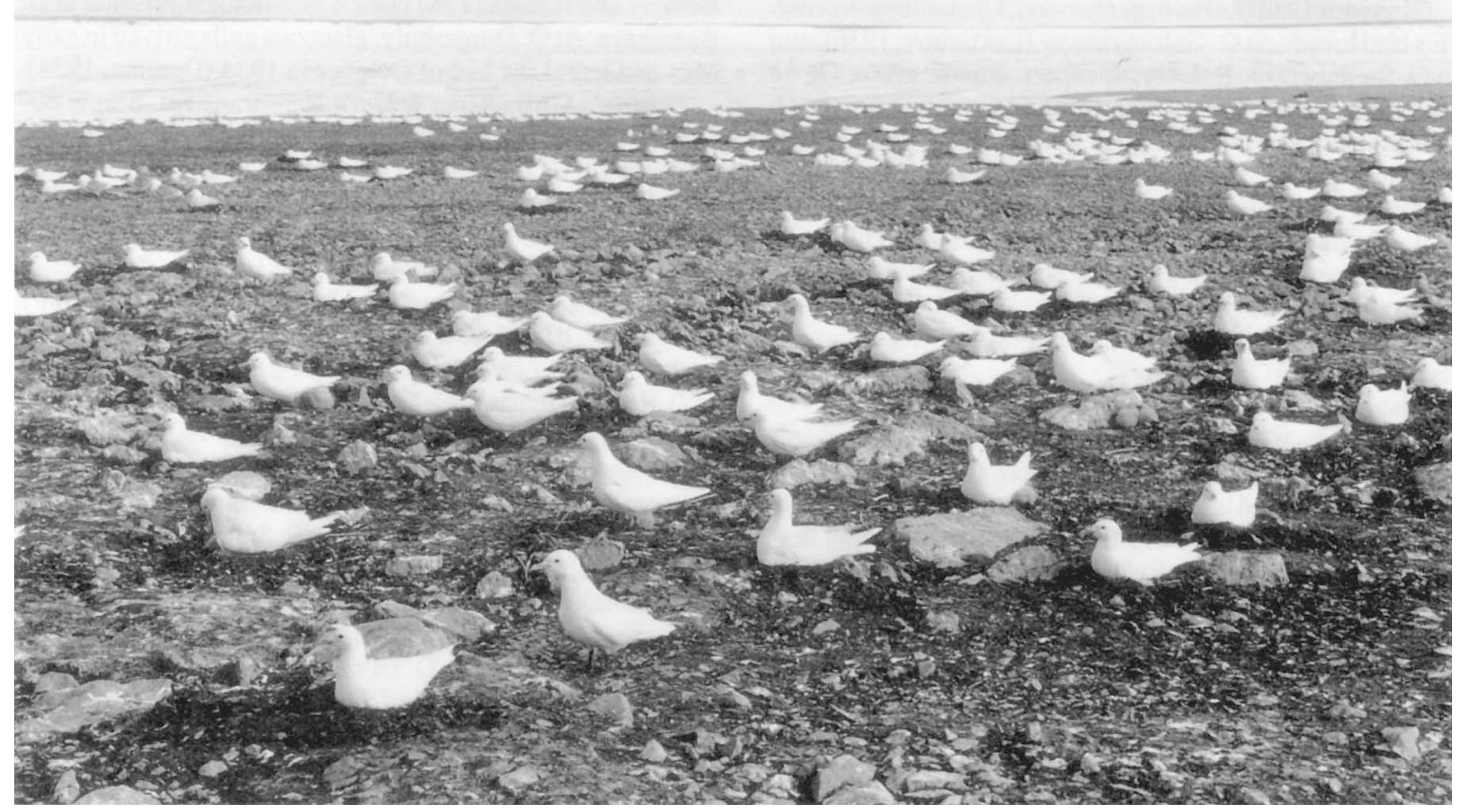

FIG. 3. Colony of ivory gulls on O. Domashniy on 15 July 1993. (Photo: J. de Korte).

kittiwakes at the head of F. Partizanskiy with some young of up to five days old. In R. Ostantsovaya, about $3 \mathrm{~km}$ from the sea, we found in 1992, 1993 and 1994 two colonies with a total of about 80 breeding pairs. We saw no young on 21 July 1994 and on 24 July 1992, but on 28 July 1992 we noticed some. In a small canyon at the western side of the southern part of Z. Ahkmatova, we found a colony of about 240 pairs in July 1994. Colonies of 200 to 300 birds are also reported from R. Tora and R. Skalistaya with no young yet on 20 July 1982 (Bulavintsev, 1984).

The earliest recorded observation is 18 April 1933 from O. Domashniy (Demme, 1934). At G. Bazarnaya, the kittiwakes had not arrived yet on 8 April 1931, but they were seen near M. Arktichesky on 13 May (Ushakov, 1951). Large groups were migrating near M. Olovyannyy on 17 May 1936 (Uspenskiy, 1969). The latest recorded observations on O. Domashniy are from the second half of September in 1930, 1931, 1932 and 1949, and from 21 October in 1950 (Demme, 1934; unpubl. logbooks, Polar Station 1937 and 1949; Uspenskiy, 1969). At G. Bazarnaya a few were still present on 17 September 1983 (Belikov and Randla, 1987).

Ivory Gull (Pagophila eburnea): Common breeder. On O. Golomyannyy, O. Domashniy, O. Sredniy (and probably O. Dlinnyy), ivory gulls breed in small and large colonies of up to 1100 breeding pairs on flat ground (Fig. 3). The colonies change in size and location between years (Demme, 1934; Urvantsev, 1935; Laktionov, 1936; Ushakov, 1951;
Uspenskiy, 1969; Semenov, 1971; de Korte and Volkov, 1993). On O. Golomyannyy, eggs were laid in June 1939, when 300 eggs were consumed by the crew of the station. (Kharitonovitch, 1940). On O. Domashniy, the first eggs were laid on 23 June 1948 and 28 June 1951 (unpubl. logbooks, Polar Station), and 11 July 1933 (Demme, 1934). Young were seen by us on O. Domashniy on 15 July 1993, when $30 \%$ of about 700 breeding pairs had young; on 22 July $70 \%$ had young. On 19 July 1994, this colony had about 1100 breeding pairs, of which about $10 \%$ had young less than one week old. About $15 \%$ had one-egg clutches, $70 \%$ had twoegg clutches and $15 \%$ had three-egg clutches. On O. Golomyannyy, flying young of the year were seen on 17 August 1931 (Ushakov, 1951), but in 1934 they still could not fly on 28 August (Demme, 1934). Belikov and Randla (1987) report ivory gulls from B. Skrytaya, but in 1993 we found only glaucous gulls and kittiwakes there. Laktionov (1936) reports nesting at O. Diabazovye, and Semenov (1971) at M. Goristyy and G. Bazarnaya, but in 1993 we did not notice them. We did, however, see a small gull colony at the north side of F. Matusevicha, on a mountain some way inland. In 1985 two pairs were found on the rocks just south of B. Skazochnaya. The existence of a colony on P. Parizhskoy Kommuny (Laktionov, 1936) was not confirmed by Belikov and Randla (1987). We saw in 1993 and 1994 a small colony on the north side of G. Ploskaya. In the river of the canyon from Oz. Izmenchivoe a group of about 30 , mainly adult birds, was 
observed on 7 September 1983 (Belikov and Randla, 1987). Breeding has been reported on O. Bol'shoy in 1956 (unpubl. logbook, Polar Station). According to Demme (1934) and Laktionov (1936), ivory gulls breed on M. Massivnyy and according to Ushakov (1951) and Urvantsev (1935), they breed on M. Olovyannyy, but we did not observe colonies there in 1993 and 1994. The report of Belikov and Randla (1987) about breeding in R. Studenaya and M. Peschannyy was not confirmed by Volkov and Pridatko (1994) in 1991 or in 1992, 1993 and 1994. In 1991, some kilometres east of R. Ostantsovaya, Volkov and Pridatko (1994) found a colony with 18 nests with an average clutch size of 16 eggs, of which $70 \%$ of the young had hatched on 28 July, and a single nest near Oz. Tverdoe, all of them on flat ground. Near L. Voytsekhovskogo they found on accessible cliffs about 50 breeding pairs, of which $90 \%$ of the young had hatched on 7 August. In 1992 all these sites were deserted, but on 21 July 1994 again about 30 pairs bred near L. Voytsekhovskogo. In the valley of R. Mushketova we found in 1992 an old empty nest. On cliffs in the canyon of R. Ostantsovaya on three sites about $1 \mathrm{~km}$ apart we found a total of 15 breeding pairs in 1992 and 1994, though the birds had deserted one site in 1994. On 28 July 1992 we saw young in these colonies for the first time. In the valley of R. Shumnaya, $8 \mathrm{~km}$ from the coast, Bulavintsev (1984) describes a colony with about 40 nests on flat ground in 1982. One $\mathrm{km}$ from this colony he found an area with about 40 old deserted nests. On O. Malyy Taymyr, A.B. Tyuryakov (pers. comm. 1988) reports a breeding place on flat ground, but in 1993 we did not observe it.

On O. Domashniy the earliest recorded arrival dates are 12 April 1951 and 22 April 1950 (unpubl. logbooks, Polar Station). Nests were still empty on 19 July 1931 at M. Olovyanny (Ushakov, 1951). The latest recorded observations from 1947 until 1951 are from the second half of October, and apply to juvenile birds (unpubl. logbooks, Polar Station).

Arctic Tern (Sterna paradisaea): Occasional breeder. On P. Parizhskoy Kommuny, Belikov and Randla (1987) found a pair with a single young on 10 August 1983. Near M. Zub, Volkov and Pridatko (1994) found a one-week-old chick on 12 August 1991. By 6 August 1992 two young of a pair had hatched near B. Amba. We found at the same place a nest with two pecked eggs on 24 July 1993, and one alarm-calling pair on 21 July 1994. Near B. Solnechnaya some were nesting in 1982 (Bulavintsev, 1984).

Little Auk (Alle alle): Locally Abundant breeder. Breeding on M. Karla Libknekhta and on O. Machtovyy, O. Diabazovye, M. Voroshilova and M. Olovyannyy, reported by Demme, (1934) and Ushakov (1951) was not confirmed in 1993. Colonies of some thousands of birds are on M. Goristyy (Semenov, 1967; Belikov and Randla, 1987). In 1993 we observed little auks breeding on the west side of south F. Matusevicha and on G. Mira and probably on the west side of B. Krasnaya. On several places on G. Bazarnaya and along the east side of north and middle B. Skazochnaya there are colonies of some thousands (Ushakov, 1951; our observations, 1985, 1993, 1994). Breeding in F. Tel'mana reported by Belikov and Randla (1987) was not confirmed in 1991
(Bulavintsev, pers. comm. 1991), and in 1992, 1993 and 1994. On 18 April 1931, there were several flocks at G. Bazarnaya and on 15 May in the polynya near M. Arkticheskiy; on 9 June thousands of birds were seen on G. Bazarnaya (Urvantsev, 1935; Ushakov, 1951). Volkov and Pridatko (1994) obtained a dead bird from May 1991 near S. Prima. On 6 August 1985, some thousands were present on G. Bazarnaya (Gavrilo, 1988b). During autumn they appear in A. Sedova (Ushakov, 1951).

Brünnich's Guillemot (Uria lomvia): Rare transient. This species has been observed in A. Sedova (Laktionov, 1936; Semenov, 1971; E.S. Korotkevich, pers. comm. 1986). Belikov and Randla (1987), report it nesting on O. Chistikov, O. Machtoviy, O. Diabazovye, M. Goristyy, G. Bazarnaya and in F. Tel'mana, but in 1993 and 1994 we did not see any there.

Black Guillemot (Cepphus grylle): Common breeder. Some pairs breed on O. Sredniy (Demme, 1934; Belikov and Randla, 1987; our observations, 1993), O. Domashniy and O. Golomyannyy (Demme, 1934), but in some years they are absent (Laktionov, 1936). They breed on M. Budenogo (Laktionov, 1936), and on M. Karla Libknekhta (Demme, 1934). There are small colonies on O. Diabazovye, O. Machtovyy and O. Chistikov (Semenov, 1971; our observations, 1993). Colonies of up to a hundred pairs are found at M. Goristyy and in the mountains at the west side of F. Matusevicha (Laktionov, 1936; our observations, 1993), at M. Bazarnaya (Laktionov, 1936; Semenov, 1971; our observations, 1985, 1993, 1994), at both sides of B. Skazochnaya $(1985,1993,1994)$, and at the west side of B. Krasnaya (1993, 1994). A few pairs breed at M. Olovyannyy (1994), in the canyon of R. Ozernaya (E.S. Korotkevich, pers. comm. 1988), and in the canyon south of Oz. Izmenchivoe (Belikov and Randla 1987; our observations, 1992).

Breeding places of 5-30 pairs were recorded on both sides of F. Tel'mana by Belikov and Randla (1987) and by us in 1993 and 1994, between F. Spartak and M. Vise (1991, 1992, 1993, 1994), at the head of F. Partizanskiy (1991), in a cliff face along R. Bazovaya $(1991,1992)$ and in cliff faces of R. Ostantsovaya (1992, 1993, 1994). They probably also nest along the river just south of G. Zvezdina and in R. Studenaya (1992).

The earliest date of observation is 8 April 1931 at G. Bazarnaya (Ushakov, 1951). Near O. Domashniy they were observed until October in 1931, 1933, 1947 and 1950 (Demme, 1934; unpubl. logbooks, Polar Station; Uspenskiy, 1969). The latest observation is from 2 November 1948 near O. Domashniy (unpubl. logbook, Polar Station). Ushakov (1951) reported large flocks on 15 May 1931, in the open water of M. Arkticheskiy.

Snowy Owl (Nyctea scandiaca). Rare breeder. Snowy owls can be seen all over the archipelago in years with high lemming densities (Belikov and Randla, 1987). In 1931 single birds were seen at M. Arkticheskiy on 13 May, at G. Bazarnaya in early June, and a nest with six eggs was found near M. Serp i Molot on 3 June (Urvantsev, 1935). On 31 July 1950 , on the northern bank of R. Matusevitcha, a nest was found with two chicks, one of which could fly on 8 August. Adults were also observed in 1950 in central O. Oktyabr'skoy Revolutsii, on 12 June (one), 17 June (two) and 22 July (one), 
and on P. Parizhskoy Kommuny on 15 August (one) (E.S. Korotkevich, pers. comm. 1986). Six birds were seen near R. Gremyashchaya on 12 July 1974 (V.M. Makeev, pers. comm. 1986). A male was seen on 18 July 1985, a female on 20 July, and single birds on 19 and 28 August in the central part of $O$. Oktyabr'skoy Revolutsii. Single birds were seen on 22 June 1990, on P. Zhiloy and R. Podjomnaya (D.J. Bol'shiyanov, pers. comm. 1988). A single bird was seen near M. Baranova in 1987 (V.V. Baranov, pers. comm. 1988) and one was found dead near R. Bazovaya in 1991.

Rook (Corvus frugilegus): Rare transient. At the south coast of O. Bolshevik, a single rook was seen in July 1982 (Belikov and Randla, 1987).

White Wagtail (Motacilla alba): Rare transient. At M. Vatutina a juvenile was seen in the beginning of October 1983 (Belikov and Randla, 1987).

Lapland Bunting (Calcarius lapponicus): Rare breeder. Near R. Gremyashchaya, Belikov and Randla (1987) saw a flying brood on 2 September 1983. On the middle part of P. Parizhskoy Kommuny, a pair with two flying young was seen on 30 July 1985.

Snow Bunting (Plectrophenaxnivalis): Common breeder. Broods have been reported from O. Domashniy with earliest fledging on 6 July 1951 (unpubl. logbook, Polar Station). Earliest fledging in 1992 was observed on 21 July 1992 near R. Bazovaya. Nests were found at B. Skrytaya (Semenov, 1971; Belikov and Randla, 1987). Between B. Panfilovets and Pr. Krasnoy Armii, about 0.2 pairs per km were counted in the low areas, and about 4.3 pairs per $\mathrm{km}$ along rivers in optimal habitat (our observations, 1985; Belikov and Randla, 1987). Nests with young were found between 3 and 11 July 1985 near M. Vatutina: clutch size varied from four to five, and earliest fledging was on 11 July. The snow bunting breeds on O. Bol'shoy (unpubl. logbook, Polar Station, 1956). Between Pr. Shokal'skogo and Z. Akhmatova, we estimated in 1991 and 1992 about 0.5 pairs per $\mathrm{km}$ in the lowland areas. Six young near R. Bazovaya found on 16 July 1992 had not fledged yet by 22 July. Near B. Solnechnaya, Bulavintsev (1984) found about 0.4 pairs per $\mathrm{km}$ along the rivers.

The earliest recorded arrival dates at $\mathrm{O}$. Domashniy are 14 April 1990 and 27 April 1952 (D.J. Bolshiyanov, pers. comm. 1990; unpubl. logbook, Polar Station). The latest autumn observations at O. Domashiniy were made on 23 September 1950, 21 September 1932, and 20 September 1951 (unpubl. logbooks, Polar Station; Demme, 1934).

\section{Non-avian Predators and Prey}

The Arctic fox (Alopex lagopus) has denning areas in the valleys of R. Gremyashchaya and R. Pod'emnaya (our observations, 1985; Belikov and Randla, 1987). In 1976-77 foxes were numerous, with litters of six young, and about 300 were caught at B. Solnechnaya, but in 1982-83 they were rare. Volkov and Pridatko found a dead specimen near M. Vise in 1991. We saw one in July 1992 near R. Ostantsovaya and one in July 1993 in A. Sedova. In July 1994 we found a dead fox at G. Bazarnaya.
The wolf (Canis lupus) sometimes occurs in groups of up to three on O. Bol'shevik and O. Oktyabr'skoy Revolutsii. (Belikov and Randla, 1987). A dead wolf was found near M. Vise in July 1991.

The ermine (Mustela erminea) was often seen near the buildings at B. Solnechnaya at the end of the 1970s (Belikov and Randla, 1987). A mummified specimen was found in July 1992 near R. Bazovaya (I.N. Safronova, pers. comm. 1992).

The Arctic hare (Lepus timidus) is reported from $\mathrm{R}$. Studenaya (Belikov and Randla, 1987; our observations, 1993). In May 1990 two were seen at M. Vise (A. Yunak, pers. comm. 1992). In July 1991 we saw one near B. Amba, and in July 1992 three together on the coast of Z. Akhmatova.

The collared lemming (Dicrostonyx torquatus) occurs on all the big islands of the archipelago, in valleys of rivers and lowlands with vegetation (G.N. Oblonskiy, pers. comm. 1982; Kalyakin and Belikov, 1985; Belikov and Randla, 1987). Densities on O. Domashniy varied from 20 individuals per $\mathrm{km}^{2}$ in 1933 to 500 per km² in 1934 (Laktionov, 1936). In western O. Oktyabr'skoy Revolutsii, there were many in 1975-76 and 1979-80 (Belikov and Randla, 1987). In June and July 1985 lemmings were numerous on O. Oktyabr'skoy Revolutsii, and a summer nest with young was found near M. Vatutina, where in optimal habitat 140 holes in one hectare were counted. In spring 1985 they were met in large numbers on the glaciers in the western part of this island. In 1986-87 and 1990 there were many on O. Oktyabr'skoy Revolutsii and O. Bol'shevik (A. Yunak, pers. comm. 1992). In August 1991 we found many fresh winter nests near R. Bazovaya, but only one occupied summer nest. In July 1992 and 1993, we found old winter nests in this area, but again only one summer nest. In July 1994, we saw a few fresh winter nests and summer nests between R. Ostantsovaya and Pr. Shokal'skogo.

The reindeer (Rangifer tarandus) can occasionally be seen in the archipelago. The total population is less than 100, mainly on O. Bol'shevik, in groups of three to seven, sometimes with calves (Laktionov, 1936; G.N. Oblonskiy, pers. comm. 1982; Belikov and Randla, 1987; our observations, 1991, 1992, 1993; S. Belikov, pers.comm. 1993).

\section{DISCUSSION}

The coverage of the breeding birds of Severnaya Zemlya is still relatively poor. Only the western part of O. Oktyaber'koy Revolutsii with the base at $\mathrm{M}$. Vatutina and the northern part of O. Bol'shevik with the base at S. Prima have been thoroughly surveyed by ornithologists. From our helicopter surveys in 1992 and 1993, when we flew across the whole archipelago and visited all major islands, we can conclude that these two areas are among the most vegetated areas in Severnaya Zemlya. There was also rather good coverage of G. Bazarnaya, A. Sedova and B. Solnechnaya by scientists with some interest in birds.

Little auk and ivory gull, which both can be considered as Atlantic arctic species, reach the eastern limits of their breeding distribution in Severnaya Zemlya, though a few little auks 
are breeding in the Bering Stait (Uspenskiy, 1963; Breekenridge, 1966; Blomquist and Elander, 1981; Yudin and Firsova, 1988; Stishov et al., 1991). The herring gull, which is a boreal-low arctic species, reaches in Severnaya Zemlya its northern distribution limit (see Yudin and Firsova, 1988). The Brünnich's guillemot, which breeds in large numbers in Frans Josef Land (Gorbunov, 1932; Golovkin, 1984), northern NovayaZemlya (Belopolskii, 1961; Golovkin, 1972; our observations, 1992, 1993, 1994), Eastern Taymyr (Kozlova, 1957; Rutilevskiy, 1970), the New Siberian Islands (Uspenskiy, 1963) and Wrangel Island (Stishov et al., 1991), shows a distribution gap in Severnaya Zemlya. The lack of suitable nesting habitat near the scarce areas of open water, or the lack of open water near suitable nesting places in August when the young swim away from the colonies, is probably the cause of the absence of this species.

In general the possibilities for the small cliff and crevice nesters (little auk, black guillemot, kittiwake) are confined to a few steep cliff faces in river canyons, along coasts, and on small islands. Because of the occurrence of ground predators in Severnaya Zemlya, the lack of inaccessible breeding places may be a limiting factor for breeding. Ivory gulls breed either in colonies of different size on inaccessible cliffs, occupied every year, or in large colonies on accessible places for only a few breeding seasons (de Korte and Volkov, 1993). This is probably a strategy to avoid predation in accessible places. The Arctic tern cannot escape predation by breeding in colonies on small islands, as it does in Spitsbergen when the sea ice has dispersed in August (de Korte, 1972). In Severnaya Zemlya the sea ice between islets and mainland often does not disappear at all, so Arctic foxes may have the opportunity to visit such colonies throughout the breeding season. Arctic terns breed along coasts in single pairs at very low densities. They probably lack good foraging opportunities in August, the period when the chicks are raised.

The young of the tundra-nesting species hatch in general in the second half of July, while snow bunting and Lapland bunting fledge in this same period. Colonial seabirds hatch in general also in the second half of July. The peak of hatching is in the second week of July for the glaucous gull, in the third week of July for the ivory gull, in the fourth week of July for the kittiwake and in the first week of August for the Arctic tern.

\section{ACKNOWLEDGEMENTS}

The Netherlands Marine Research Foundation and the Plancius Foundation (Amsterdam) provided grants for our field work in 1992, 1993 and 1994. The Van Tienhoven Foundation (Amsterdam) and the Willem Barentsz Foundation (Groningen) supported working visits of A. Volkov to The Netherlands. S. Belikov (Moscow) invited Volkov to take part in his 1991 expedition. The crew of the BARC-company (St. Petersburg) at S. Prima was very helpful when we worked in their area, and they organised transport for us. N. Cartwright (Alexandria, Scotland) corrected the English.

\section{REFERENCES}

BELIKOV, S.E., 1987. Chernaya kazarka na arkhipelage Severnaya Zemlya v svyazi s osvoeniem arkhipelaga [Brent Goose on the Severnaya Zemlya archipelago in connection with exploration of the archipelago]. In: Vliyanie antropogennoy transformatsii landshaftov na populyatsii nazemnykh pozvonochnykh. Chast' 2. Moskva. 118-119.

BELIKOV, S.E., and RANDLA, T.E. 1987. Fauna ptits i mlekopitaushchikh Severnoy Zemli [Fauna of birds and mammals of Severnaya Zemlya]. In: Fauna i ecologiya ptits i mlekopitaushchikh Sredney Sibiri. Moskva: Nauka. 18-28.

BELOPOLSKII, L.O. 1961. Ecology of sea colony birds of the Barents Sea. Jerusalem: Israel Programme Scientific Translations. $346 \mathrm{p}$.

BLOMQUIST, S., and ELANDER, M. 1981. Sabine's gull (Xema sabini), Ross's gull (Rhodostethia rosea) and ivory gull (Pagophila eburnea) - Gulls in the Arctic: A review. Arctic 34:122-132.

BREEKENRIDGE, W.J. 1966. Dovekie on Little Diomede Island, Alaska. Auk 83(4):680.

BULAVINTSEV, V.I. 1984. Ptitsy ostrova Bol'shevik, arkhipelag Severnaya Zemlya [Birds of Bol'shevik Island, archipelago Severnaya Zemlya]. Ornitologiya Vol. 19. Moskva: Izd. Moskovskogo Universiteta. 175-176.

DEMME, N.P. 1934. Promyslovaya fauna Severnoy Zemli [The hunting fauna of Severnaya Zemlya]. Manuscript in State Archive of Scientific-technical documentation. St. Petersburg. 120 p.

DODD, G.J. 1985. Arctic Pilot 1. Taunton: Hydrographic Department. Ministry of Defence, Great Britain. 259-263.

GAVRILO, M.V. 1988a. Kuliki ostrova Oktyabr'skoy Revolutsii (Severnaya Zemlya) [Waders of Oktyabr'skoy Revolutsii Island (Severnaya Zemlya)]. Ornitologiya Vol. 23. Moskva: Izd. Moskovskogo Universiteta. 204.

. 1988b. Ptitsy ostrova Oktyabr'skaya Revolutsiya (Severnaya Zemlya): chislennost', osobennosti rasprede leniya, recomendatsii k okhrane [Birds of Oktyabr'skoy Revolutsii Island (Severnaya Zemlya): Number, peculiarities of distribution, recommendations for protection]. In: Biologicheskie problemy Severa. Izuchenie i okhrana ptits v ekosistemakh Severa. Vladivostok: Institut Biologicheskih Problem Severa and Vsesoyoznoe Ornitologicheskoe Obshchestvo. 38-41.

GOLOVKIN, A.N. 1972. Ptich'i bazary Severa Novoy Zemli [Bird colonies of the northern part of Novaya Zemlya]. In: Osobennosti biologicheskoy produktivnosti vod bliz ptich'ikh bazarov Severa Novoy Zemli. Leningrad: Nauka. 84-91.

1984. Seabirds nesting in the USSR: Their status and protection of populations. ICBP Technical publications 2:473-486.

GORBUNOV, G.P. 1932. Ptitsy Zemli Frantsa Iosifa [Birds of Frans Jozef Land] In: Trudy Arkticheskogo Instituta 4. Leningrad. $244 \mathrm{p}$.

KALYAKIN, V.N., and BELIKOV, S.E. 1985. Kopytnyi Lemming (Dicrostonyx torquatus) na ostrovakh archipelaga Severnaya Zemlya [Collared Lemming (Dicrostonyx torquatus) at the islands of Severnaya Zemlya archipelago]. Zoologicheskii zhurnal 64(5):788-789. 
KATALOG LEDNIKOV SSSR. 1980.[Catalogue of glaciers of the USSR. Part 1]. Leningrad: Gidrometeoizdat. 79 p.

KHARITONOVICH, B.L. 1940. Na ostrove Domashnem [On Domashniy Island]. In: Sovetskaya Arktika 8. Moskva: Selkhozgiz. 60-66.

KHODACHEK, E.A. 1986. Osnovnye rastitel'nye soobshchestva zapadnoy chasti O. Oktyabr'skoy Revolutsii (Severnaya Zemlya) [The main plant communities of the western part of Oktyabr'skoy Revolutsii Island (Severnaya Zemlya)]. Botanicheskiy zhurnal 71(12):1628-1638.

KOROTKEVICH, E.S. 1958. Rastitel'nost Severnoy Zemli [The vegetation of Severnaya Zemlya]. Botanicheskiy zhurnal 43(5):644-663.

KORTE, J. DE. 1972. Birds observed and collected by Nederlandse Spitsbergen Expeditie 1967 and 1968/69, first, second and last part. Beaufortia 19(253):113 - 150, (257):197232, 20(261): $23-58$.

KORTE, J. DE, and VOLKOV, A.E. 1993. Large colony of Ivory Gulls Pagophila eburnea at Domashniy Island, Severnaya Zemlya. Sula 7(3):107-110.

KOZLOVA, E.V. 1957. Rzhankoobraznye: podotryad chistikovye [Charadriiformes; Alcidae]. Fauna SSSR 2(3). MoskvaLeningrad: Nauka. 114-143.

LAKTIONOV, A.F. 1936. Severnaya Zemlya. Arkhangel'sk: Severnoe kraevoe izdatel'stvo. 117 p.

LOGBOOKS. 1931-1952. From Polar Stations in Severnaya Zemlya. Available in the Archives of Arctic and Antarctic Research Institute, ulitsa Beringa 38, St. Petersburg, Russia.

NASONOV, N.V. 1916. Zoologicheskie kollektsii, sobrannye Gidrograficheskoy ekspeditsiey Severnogo Ledovitogo Okeanana 'Taymyre' i 'Vaygache' v 1910-1915 gg. i peredannye Zoologicheskomu muzeyu Imperatorskoy Akademii nauk [Zoological collections that were collected by Hydrographical Expedition to Arctic Ocean by 'Taymyr' and 'Vaygach' 1910 1915 and passed to Zoological Museum of Imperial Academy of Science]. Izvestiya Imperatorskoy Akademii Nauk. Seriya 6. Tom X. St. Petersburg. 1493-1504.

RUTILEVSKIY, G.L. 1970. Zhivotnyy mir [World of animals]. In: Taymyrsko-Severozemel'skaya oblast'. Leningrad: Gidrometeoizdat. 301-333.

SAFRONOVA, I.N. 1981. Flora O. Oktyabr'skoy Revolutsii [The flora of O. Oktyabr'skoy Revolutsii]. Trudy Arkticheskogo i Antarkticheskogo Insituta, 367. Leningrad. 142-151.

. 1993. O flore ostrova Bol'shevik (arkhipelag Severnaya Zemlya) [On the flora of Bol'shevik Island (Severnaya Zemlya archipelago)]. Botanicheskiy zhurnal 78(2):79-84.
SDOBNIKOV, V.M. 1960. Ptitsy Tsentral'nogo i Severnogo Taymyra i Severnoy Zemli [Birds of Central and Northern Taymyr and Severnaya Zemlya]. Unpubl. ms. Available at Department of Geography of Arctic and Antarctic Research Institute, ulitsa Beringa 38, St. Petersburg, Russia.

SEMENOV, V.I. 1967. Severnaya Zemlya. Fiziko-geograficheskaya kharakteristika. [Severnaya Zemlya. Physical-geographical characteristics]. Leningrad. 196 p.

1970. Morya omyvajushchie poluostrov Taimyr i Severnuyu Zemlyu [The seas washing the Taymyr Peninsula and Severnaya Zemlya]. Leningrad: Gidrometeoizdat. 35-62.

- 1971. Prirodnye rayony Severnoy Zemli [Natural regions of Severnaya Zemlya]. Trudy Arkticheskogo i Antarkticheskogo Instituta 304. Leningrad. 167-209.

STARKOV, V.F. 1985. Istoriya geograficheskikh otkrytiy $i$ is sledovaniy. Severo-vostochnyy morskoy prokhod [History of geographical discoveries and studies. Northeast sea route]. In: Atlas Arktiki. Moskva: GUGK. 156-158.

STAROKADOMSKIY, L.M. 1946. Ekspeditsiya Severnogo Ledovitogo Okeana, 1910-1915 [The expedition of the Arctic Ocean in 1910-1915]. Moscow and Leningrad: Izdatel'stvo Glavsemorputi. 320 p.

STISHOV, M.S., PRIDATKO, V.I., and BARANYUK, V.V. 1991. Ptitsy ostrova Vrangelya [The birds of Wrangel Island]. Novosibirsk: Nauka. 252 p.

URVANTSEV, N.N. 1935. Dva goda na Severnoy Zemle [Two years in Severnaya Zemlya]. Leningrad: Glavsevmorput'. 363 p.

USHAKOV, G.A. 1951. Po nekhozhenoy zemle [On untouched land]. Moskva-Leningrad: Glavsevmorput'. 393 p.

USPENSKIY, S.M. 1963. Ptitsy i mlekopitaushchie ostrova Benneta [Birds and mammals of Benneta Island]. In: Trudy Arkticheskogo i Antarkticheskogo Instituta 224. Leningrad: "Morskoy transport". 180-205.

. 1969. Zhizn' v vysokikh shirotakh na primere ptits [Life in high latitudes, with birds as an example]. Moskva: Mysl'. 463 p.

VOLKOV, A.E., and PRIDATKO, V.I. 1994. Materialy po faune i naseleniyu ptits severno-zapadnoy chasti ostrova Bol'shevik (arkhipelag Severnaya Zemlya) [Data on fauna and bird communities of the northwestern part of Bol'shevik Island (Severnaya Zemlya archipelago)]. In: Arkticheskie tundry Taymyra i ostrovov Karskogo morya: Priroda, zhivotnyy mir i problemy ikh okhrany. Moskva: Nauka. 149-157.

VOOUS, K.H. 1977. List of recent Holarctic bird species. London: British Ornithologists' Union. 85 p.

YUDIN, K.A. and FIRSOVA, L.V. 1988. Belaya chayka [Ivory Gull], Serebristaya chayka [Herring Gull]. In: Ptitsy SSSR. Chaykovye. Moskva: Nauka. 51-57, 126-146. 